\title{
Special Aspects in Attitude Control of a Spacecraft, Equipped With Inertial Actuators
}

\author{
Levskii MV* \\ Research Institute of Space systems, Khrunichev State Space research-and-production Center, Korolev, Moscow region, Russia
}

Received: September 19, 2017; Accepted: October 09, 2017; Published:October 31, 2017

*Corresponding author: Levskii MV, Research Institute of Space systems, Khrunichev State Space research-and-production Center, Korolev, Moscow region, Russia, E-mail:dp940@mail.ru

\begin{abstract}
The problem of correct choosing the time of optimal rotation from an arbitrary initial position into prescribed final angular position is investigated. The case, when slew maneuver is carried out with minimal magnitude of spacecraft angular momentum, is considered. Calculated dependences for constructing optimal program of reorientation (in sense of minimum angular momentum) are written as the law of variation of the angular velocity vector. Optimal control is in the class of regular motions. Dynamics of spacecraft rotation during the slew maneuver is similar to the known method of control, designed earlier, which includes as much as possible fast acceleration of a spacecraft, rotation with constant modulus of angular momentum and as much as possible fast cancellation of angular momentum. The formalized equations are presented, and computational expressions for determination of optimal duration of reorientation maneuver are obtained for known mass-inertial characteristics of a spacecraft, if attitude control is made by inertial actuators (system of control moment gyroscopes, gyrodynes). The knowledge of fit range of preferable duration of a slew maneuver helps to plan correctly the flight program of spacecraft controlled by the powered gyroscopes. The condition for determining the moment of the beginning of deceleration which uses current parameters of motion (information on angular position of a spacecraft and measurements of angular velocity) was given, what considerably increases accuracy of spacecraft move into a required position. Example of optimal spacecraft rotation and results of mathematical simulation adds the made theoretical descriptions, and illustrates reorientation process in visual form.
\end{abstract}

Keywords: Optimal control; Spatial orientation; Spacecraft attitude; Optimized functions; Control moment gyroscopes;

\section{Introduction}

Below we solve the problem of a choosing the duration of maneuver of spacecraft transfer into position of the required orientation (reorientation maneuver of a spacecraft). As spatial reorientation we mean transfer of axes OXYZ bounded to the spacecraft body from one known angular position into another known (typically prescribed) angular position in a finite amount of time T. In this case, parameters of a slew maneuver (for example, components of slew quaternion) are known in advance, even before the beginning of maneuver; any initial angular mismatches can be possible (from a few degrees up to $180^{\circ}$ ). The angular orientation of the right-hand coordinate system OXYZ (as well as its initial and final positions, $O X_{\mathrm{st}} Y_{\mathrm{st}} Z_{\mathrm{st}}$ and $O X_{\mathrm{f}} Y_{\mathrm{f}} Z_{\mathrm{f}}$ ) is determined relative to a chosen coordinate system (reference basis I). In most cases the reference basis is inertial coordinate system $O X_{\text {in }} Y_{\text {in }} Z_{\text {in }}$ (ICS).

Many papers are dedicated to questions of controlled rotations of solid body around its centre of mass [1-13]. In particular, the kinematic problem of a slew maneuver is studied in detail where solution was resulted for variant when angular velocity vector is limited by modulus [3]. In [4], the questions of optimal rotation of a spacecraft by criteria of fast response and minimum of energy expense for a case when the region of admissible values of the controlling moment is limited by sphere are considered, and itself spacecraft rotates around the vector of finite slew. Interesting results are presented in [5] where control is constructed by method of the combined synthesis by criterion of the generalized work. Later works $[6,7]$ are known also (including decisions on the basis of algorithms of indistinct logic [7]) which also describe the constructing the controls stabilizing rotation of a spacecraft abound the instantaneous Euler axis (we will notice, that the majority of decisions corresponds to spacecraft rotation around motionless axis) though the rotation in the plane of the least slew angle is not always optimum in practice (but only in individual special cases), however exactly it was not executed [3-8].

In this paper, it is supposed that inertial actuators, in particular, powered gyroscopes (or gyrodynes), are used for control of spacecraft attitude, and size of angular momentum of spacecraft becomes essential-critical, therefore we must take into account this size at control of spacecraft rotation [9]. Earlier, the control method for spacecraft reorientation has been designed, which takes into account restriction of angular momentum of a spacecraft [1]. This method is accepted as model motion (as reference). From theoretical studies is known that level of spacecraft's angular momentum and time of a slew maneuver $T$ are interdependent; if angular momentum is small (i.e. less) then duration of maneuver is more large $[1,2,12]$. However at presence of perturbations acting upon the spacecraft, the choice of time $T$ is not so obvious (further we will show that available stock of the angular momentum of gyro-system has significant role at timing T). This investigation is dedicated to finding of optimal duration of spacecraft reorientation (within the mode [1]) at which the reserved stock of angular momentum of gyroscopes system would be maximum. 


\section{Equations of motion and statement of the control problem}

It is assumed that the spacecraft attitude control is realized using actuators that generate torques relative to all three principal central axis of inertia of the spacecraft. Angular motion of the spacecraft as a solid body we describe by the equations written down in quaternion variables [3].

$$
2 \dot{\Lambda}=\Lambda \circ \omega
$$

where $\omega$ is the vector of absolute angular velocity of a spacecraft; $\Lambda$ is the quaternion reflected spacecraft orientation relative to inertial basis $\boldsymbol{I}$, and the symbol $\circ$ denotes the multiplication of quaternion's. The equation (1) has the boundary conditions $\Lambda(0)=\Lambda_{\text {in }}$ and $\Lambda(T)=\Lambda$ f. The quaternions $\Lambda \mathrm{in}$ and $\Lambda \mathrm{f}$ that specify directions of spacecraft axes at initial and final moments of time have any arbitrary a priori given values satisfying the condition $\|\Lambda \mathrm{in}\|=\|\Lambda \mathrm{f}\|=1$ Spacecraft motion is considered optimum if the value

$$
\max _{0<t<T} \sqrt{J_{1}^{2} \omega_{1}^{2}+J_{2}^{2} \omega_{2}^{2}+J_{3}^{2} \omega_{3}^{2}}
$$

is minimal ( $\mathrm{T}$ is the time of termination of the slew maneuver), where $\omega_{i}$ are the projections of the absolute angular velocity on axes of the body's coordinate system, $J_{i}$ are the spacecraft central principal moments of inertia. Optimal control of a spatial rotation consists in transfer of a spacecraft from the position $\Lambda_{\text {in }}$ into the position $\Lambda_{\mathrm{f}}$ according to the equation (1) with the minimum value of the index (2) which corresponds to maximal magnitude of angular momentum $\mathrm{L}$ with the components $\mathrm{L}_{\mathrm{i}}=\mathrm{J}_{\mathrm{i}} \omega_{i}$.

For the spacecrafts, controlled by powered gyroscopes (gyrodynes or flywheels), minimization of angular momentum of a spacecraft body is very important. Now powered gyroscopes (gyrodynes) have received good application as executive mechanisms of spacecraft attitude system. Their use in regime of a slew maneuver demands that the summary angular momentum of the gyro-system would not exceed the admissible value. At control of spacecraft attitude with use of inertial actuators (control moment gyroscopes), the vector of angular momentum of the gyro-system should be within preset limited region $S$, and the extending of this vector beyond the region $S$ leads to loss of controllability of a spacecraft; the size of available angular momentum determines the controlling possibilities of gyrosystem. Control of spacecraft rotation is a chieved by redistribution of the angular momentum between the system of gyrodynes and spacecraft body; total angular momentum of a spacecraft as solid body with rotating masses is equal or close to zero [9]. When designing, analyzing, elaborating, and modeling the algorithms of attitude control of a spacecraft with powered gyroscopes it is assumed that region $\mathrm{S}$ of available angular momentum of the system of powered gyroscopes is limited by a sphere with radius $\mathrm{R}_{0}$. Such statement is used by many developers, designers and researchers [14-17]; it is valid for a large number (if not for the majority) of spacecraft. Obviously, for the guaranteed presence of angular momentum $\mathrm{G}$ of powered gyroscopes's system within region $\mathrm{S}$ it is necessary that | $\mathbf{G}$ | was as small as possible. Hence, it is necessary, that during operated rotation of a spacecraft from position $\Lambda_{\text {in }}$ in position $\Lambda_{\mathrm{f}}$ the modulus of angular momentum of spacecraft body was minimum possible (since $\mathbf{L}+\mathbf{G} \approx 0$, where $\mathrm{L}$ is the angular momentum of the spacecraft body, and $\mathrm{G}$ is the angular momentum of the system of powered gyroscopes). From here we understand a sense of the formulated problem of control with minimization of the index (2), because the stock of the angular momentum of the gyro-system, determined by difference $\mathrm{R}_{0}-|\mathrm{L}|$ will be maximal in this case only.

\section{Formalization of optimal control of spacecraft's slew maneuver}

Let us shortly write down basic relationships and equations describing optimal motion of a spacecraft. The accepted functional (2) does not contain (in an explicit form) components $M_{i}$ of the moment of forces. Therefore the projections of angular velocity $\omega_{i}(i=\overline{1,3})$ are considered as the controlling variables (controls).

It is known the following. Minimal value of index (2) is no less than quantity $F / T$, where

$$
F=\int_{0}^{T} \sqrt{J_{1}^{2} \omega_{1}^{2}+J_{2}^{2} \omega_{2}^{2}+J_{3}^{2} \omega_{3}^{2}} d t
$$

But index (2) is equal to $F / T$ if angular velocity $\omega$ is piecewise continuous function (i.e. when the jump-like changes in the angular velocity are possible and the situation $\omega(0+) \neq 0$, $\omega(T) \neq 00$ is admissible) and $|\mathrm{L}|=$ const within interval of time $0<$ $\mathrm{t}<\mathrm{T}$. We must minimize the integral $\mathrm{F}$ above all. For minimization of the integral $\mathrm{F}$, the Hamilton function

$$
H=0.5 r_{0}\left(\omega_{1} p_{1}+\omega_{2} p_{2}+\omega_{3} p_{3}\right)-\sqrt{J_{1}^{2} \omega_{1}^{2}+J_{2}^{2} \omega_{2}^{2}+J_{3}^{2} \omega_{3}^{2}}
$$

May be used together with the equations [3]

$$
\dot{p}_{1}=\omega_{3} p_{2}-\omega_{2} p_{3}, \dot{p}_{2}=\omega_{1} p_{3}-\omega_{3} p_{1}, \dot{p}_{3}=\omega_{2} p_{1}-\omega_{1} p_{2}(3)
$$

Where $p_{1}, p_{2}, p_{3}$ are projections of certain vector $\mathbf{p}$ onto axes related with spacecraft, and $|\mathrm{P}|=1 ; \mathrm{r}_{0}=$ const $>0$ (the equations (3) show that the vector $\mathbf{p}$ is immovable relative to inertial basis I).

If there were no restrictions of the moment of forces, then optimal rotation of a spacecraft (in sense of criterion (2)) satisfies the equations (3) and the equations [2]

$$
\omega_{i}=b p_{i} / J_{i}^{2}
$$

Where $\mathrm{b}>0$ is scalar value. Note that $T \neq 0$ (the time $\mathrm{T}$ satisfies the condition $0<T<\infty$ ). It is obvious that the equations (4) provide maximum of the Hamiltonian $\mathrm{H}$ for any $b$. Optimal value is

$$
r_{0}=1 / \sqrt{p_{1}^{2} / J_{1}^{2}+p_{2}^{2} / J_{2}^{2}+p_{3}^{2} / J_{3}^{2}}
$$

Since the turn is terminated at time $T$, i.e. the terminal condition $\Lambda(T)=\Lambda$ f must be satisfied at given time $T$ ). The problem of determining the optimal control consists in solving 
the system of equations of angular motion (1) and the equations (3) under the condition that optimal vector-function $\omega(t)$ is chosen basing on the requirements (4) and this solution satisfies the boundary conditions $\Lambda(0)=\Lambda_{\text {in }}, \Lambda(T)=\Lambda_{\mathrm{f}}$. The angular momentum $\mathbf{L}$ and the magnitude $b$ are connected by the formula:

$$
\mathbf{L}^{2}=b^{2}\left(p_{1}^{2} / J_{1}^{2}+p_{2}^{2} / J_{2}^{2}+p_{3}^{2} / J_{3}^{2}\right)
$$

Notice that $p_{1}^{2} / J_{1}^{2}+p_{2}^{2} / J_{2}^{2}+p_{3}^{2} / J_{3}^{2}=$ const for system of the equations (3), (4) (since $p_{1} \dot{p}_{1} / J_{1}^{2}+p_{2} \dot{p}_{2} / J_{2}^{2}+p_{3} \dot{p}_{3} / J_{3}^{2}$ $=0$ for values $p_{i}$ calculated using the equations (3) with $\omega_{i}$ calculated according to (4)). In case of unlimited moments $M_{i}$, spacecraft rotates with $|\mathbf{L}|=$ const $=F / T$ at entire interval of motion $0<t<T$; optimal rotation of a spacecraft is carried out with constant modulus of angular momentum $L_{m}$. Concrete value $L_{m}$ is unequivocally determined by time of maneuver termination $T$. The optimal vectors $\omega$ and $\mathbf{p}$ are connected by relation [2]

$$
\omega_{i}=\frac{L_{m} p_{i}}{J_{i}^{2} \sqrt{p_{1}^{2} / J_{1}^{2}+p_{2}^{2} / J_{2}^{2}+p_{3}^{2} / J_{3}^{2}}}(i=\overline{1,3})
$$

At satisfying the conditions $\Lambda(0)=\Lambda_{\text {in }}, \Lambda(T)=\Lambda_{\mathrm{f}}$ for solution to equations (1) $\Lambda(t)$, where $L_{m}>0$ is the magnitude (modulus) of angular momentum with which the slew maneuver of a spacecraft is made. The equations for angular velocities $\omega_{i}$ can be formalized in following form:

$$
\begin{gathered}
\dot{\omega}_{1}=\omega_{2} \omega_{3}\left(J_{2}^{2}-J_{3}^{2}\right) / J_{1}^{2}, \dot{\omega}_{2}=\omega_{1} \omega_{3}\left(J_{3}^{2}-J_{1}^{2}\right) / J_{2}^{2}, \\
\dot{\omega}_{3}=\omega_{1} \omega_{2}\left(J_{1}^{2}-J_{2}^{2}\right) / J_{3}^{2}
\end{gathered}
$$

During optimal maneuver (without restrictions for Mi ), the desired solution $\boldsymbol{\omega}(\mathrm{t})$ has the following properties

$J_{1}^{2} \omega_{1}^{2}+J_{2}{ }^{2} \omega_{2}{ }^{2}+J_{3}{ }^{2} \omega_{3}{ }^{2}=R=$ const,$J_{1}{ }^{4} \omega_{1}{ }^{2}+J_{2}{ }^{4} \omega_{2}{ }^{2}+J_{3}{ }^{4} \omega_{3}{ }^{2}=D=$ const

Optimal control of spatial rotation consists of a short-term imparting the initial conditions of motion (the calculated angular velocity) to the spacecraft at the beginning of a slew maneuver, maintenance of spacecraft rotation with demanded (programmed) angular velocity $\boldsymbol{\omega}(t)$ at which the modulus of angular momentum of a spacecraft has constant value $|\mathbf{L}|=$ const, and a short-term suppressing of available angular velocity to zero at the moment of time $t=T$, when $\Lambda(t)=\Lambda_{\mathrm{f}}$ (at achievement by spacecraft of final position $\Lambda \mathrm{f}$ ). Key problem is the finding the law of variation of the vector $\mathbf{p}(t)$ and value $L_{m}$ that at result of solving the system of the equations (1), (3), (5) with the initial condition $\Lambda(0)=\Lambda_{\text {in }}$, the boundary condition $\Lambda(T)=\Lambda_{\mathrm{f}}$ was satisfied at right endpoint (the determination of the vector $\mathbf{p}(0)$ is a separate and rather complicated problem).

The problems in which the boundary values $\omega(0)=\omega(T)=0$ (such conditions of spacecraft rotation are most typical) are of practical importance. Of course, at the times $t=0$ and $t=T$ the angular velocity for a nominal program of spacecraft rotation, determined by equations (5), are not equal to zero. Consequently, transferring segments are unavoidable: acceleration of rotation as a transfer from the state of rest (when $\omega=0$ ) to the regime of rotation with an angular momentum of maximal magnitude $H_{0}$, and braking, i.e., reduction of the spacecraft's angular momentum down to zero; and for that to finish a maneuver during given time $T$ should be $H_{0}>L_{m}$. Between acceleration of rotation and braking, the equations ( 3 ) and

$$
\omega_{i}=\frac{H_{0} p_{i}}{J_{i}^{2} \sqrt{p_{1}^{2} / J_{1}^{2}+p_{2}^{2} / J_{2}^{2}+p_{3}^{2} / J_{3}^{2}}}\left(i=\overline{1,3}, \mathrm{H}_{0}=\text { Const }\right)
$$

are satisfied.

If the conditions of slew maneuver $\Lambda_{\text {in }}, \Lambda_{\mathrm{f}}$, and the time $T$ are such that times of acceleration and braking are very small (in comparison with the total time of a maneuver $T$ ) and we may to neglect them, then one can consider as impulsive processes both imparting necessary angular momentum $H_{0}$ to the spacecraft and reducing available angular momentum down to zero, and almost during the entire maneuver (between acceleration and braking) $|\mathbf{L}(t)|=$ const $=H_{0}$ with satisfaction of the equations (3), (8). When finding optimal solutions $\mathbf{p}(t), \omega(t)$ the value of vector $\mathbf{p}$ at the moment of instant $t=0$ is determinative.

If the controlling moment $\mathrm{M}$ is limited, then a boost of spacecraft angular momentum to the required level $|\mathbf{L}|=H_{0}$ at the beginning of the slew maneuver and damping of available angular momentum to zero at the end of reorientation maneuver occupy some finite (distinct from zero) time. Of interest is the general case, when conditions of slew maneuver $\Lambda$ in and $\Lambda \mathrm{f}$ are such that one cannot neglect the transition segments (acceleration and braking). Let the vector $\mathbf{M}$ satisfy the condition

$$
M_{1}^{2}+M_{2}^{2}+M_{3}^{2} \leq m_{0}^{2}
$$

Where $\boldsymbol{M}_{\boldsymbol{i}}$ are the projections of the moment of forces $\mathrm{M}$ on the central principal axes of the spacecraft; $\boldsymbol{m}_{\boldsymbol{0}}$ is the maximum admissible magnitude of the controlling moment. Dynamics of spacecraft rotation is described by the equations

$$
\begin{gathered}
J_{1} \dot{\omega}_{1}+\left(J_{3}-J_{2}\right) \omega_{2} \omega_{3}=M_{1}, J_{2} \dot{\omega}_{2}+\left(J_{1}-J_{3}\right) \omega_{1} \omega_{3}=M_{2} \\
J_{3} \dot{\omega}_{3}+\left(J_{2}-J_{1}\right) \omega_{1} \omega_{2}=M_{3} \text { (10) }
\end{gathered}
$$

The laws of the fastest imparting and reduction of the angular velocity under the constraint (9) are known [10]. At the segment of acceleration, optimal control has the form

$$
\mathbf{M}=m_{0} J . \omega /|J . \omega|,
$$


Where $\mathrm{J}=\operatorname{diag}(\mathrm{J} 1, \mathrm{~J} 2, \mathrm{~J} 3)$ is the inertia tensor of spacecraft. At optimal motion, angular momentum of a spacecraft does not change the direction in inertial coordinate system, because the controlling moment $\mathrm{M}$ and angular momentum $\mathrm{L}$ have same direction. The magnitude of angular momentum varies according to the law $|\mathbf{L}|=m_{0}$ t. At the segment of braking, optimal control we write in following form

$$
\mathbf{M}=-m_{0} J . \omega /|J . \omega|(12)
$$

At optimal motion, angular momentum of a spacecraft does not change the direction in inertial coordinate system, and the controlling moment $\mathrm{M}$ makes with the angular momentum an angle of 180 degree. The angular momentum varies according to the law $\mathbf{L}=\operatorname{Lbr}-m_{0}\left(t-t_{0}\right)$, where $L \mathrm{br}=\left|J \cdot \omega\left(t_{0}\right)\right| ; t_{0}$ is time of beginning of rotation damping. For both acceleration and braking, optimal control (as fast response) is control under which the controlling moment is parallel to angular momentum of a spacecraft at any moment of time.

At time instant $t=0$ the angular momentum of a spacecraft $\mathbf{L}=0$, and the control (11) is necessary for the fastest reaching the preset level $\mathbf{L}=H_{0}$. Until $|J \omega(t)|<H_{0}$, the controlling moment $\mathbf{M}=m 0 \mathbf{L} /|\mathbf{L}|$ is optimum. Since the instant tac when the equality $|J \omega(\operatorname{tac})|=H_{0}$ becomes valid, optimum will be the motion (3), (8) at which the equality $|J \omega(t)|=H 0$ take place. It is obvious that because of existence of the boundary condition $\omega(T)=0$ such a time instant $t \mathrm{br}\left(t_{\mathrm{br}}<T\right)$ should exist starting from which the angular momentum is begun to be reduced at a maximum controlling moment $\mathrm{M}=-m_{0} \mathbf{L} /|\mathbf{L}|$ (the instant $t_{\mathrm{br}}$ is chosen so that by the moment of a absolute stop $\omega=0$ the spacecraft would occupy the desired angular position $\Lambda_{\mathrm{f}}$ ). At the intervals of rotation acceleration and braking, the controlling moment $\mathbf{M}$ has ultimate maximum, and condition (9) is a strict equality, and within interval between acceleration and braking, the equations (3), (8) and equality $|\mathbf{L}|=$ const $=H_{0}$ are valid. As result, the trajectory of spacecraft rotation $\Lambda(t)$ is partitioned in three components: $\Lambda(0)-\Lambda\left(t_{\mathrm{ac}}\right), \Lambda\left(t_{\mathrm{ac}}\right)-\Lambda\left(t_{\mathrm{br}}\right)$, and $\Lambda\left(t_{\mathrm{br}}\right)-\Lambda(T)$. Let us represent the quaternion of a slew in the form:

$$
\Lambda_{\mathrm{s}}=\tilde{\Lambda}_{\text {in }} \circ \Lambda_{\mathrm{f}}=\Delta \Lambda_{\mathrm{ac}} \circ \Delta \Lambda_{\text {nom }} \circ \Delta \Lambda_{\mathrm{br}},
$$

Where $\Delta \Lambda_{\mathrm{ac}}=\tilde{\Lambda}_{\mathrm{in}} \circ \Lambda\left(t_{\mathrm{ac}}\right)$ is the quaternion of spacecraft rotation during the acceleration segment; $\Delta \Lambda_{\mathrm{br}}=\tilde{\Lambda}\left(t_{\mathrm{br}}\right) \circ \Lambda_{\mathrm{f}}$ is the quaternion of spacecraft rotation during braking; $\Delta \Lambda_{\text {nom }}=\tilde{\Lambda}\left(t_{\text {ac }}\right) \circ \Lambda\left(t_{\text {br }}\right)$ is the quaternion of rotation during spacecraft rotation with maximum angular momentum $\mathrm{H}_{0}(\tilde{\Lambda}$ is the conjugate of the quaternion $\Lambda$, and $\tilde{\Lambda}_{\text {in }}$ is the conjugate of the quaternion $\Lambda_{\text {in }}$ ).

The optimal motion of a spacecraft consists of segments on which the controlling moment maximum in magnitude acts (segments of acceleration and braking), and of a segment of rotation with constant (in modulus) angular momentum, equal to the designed value $H_{0}$ supporting the reaching of final position
$\Lambda_{\mathrm{f}}$ in the given time $T$. On the segment of maximal controlling moment the angular momentum vector $\mathrm{L}$ has a permanent direction in the inertial space, but it is variable in magnitude (increase up to present value on the acceleration segment, and decrease to zero on the braking segment), while the controlling moment $\mathbf{M}$ is immovable with respect to the reference basis $\boldsymbol{I}$ (the vectors $\mathbf{M}$ and $\mathbf{L}$ are parallel); angular motion is determined by equations (1), (10). During the spacecraft rotation with maximum angular momentum the parameters of motion are determined by equations (3), (8). In this case, the angular momentum vector $\mathrm{L}$ has a constant magnitude $\mathrm{H}_{0}$, but its direction varies from a position preset at spacecraft acceleration to a position required during spacecraft braking. The spacecraft motion during its slew maneuver proceeds according to the following program of angular momentum variation: an increase of the magnitude of vector $\mathrm{L}$ from zero to $\mathrm{H}_{0}$ with a maximum velocity (with maximum moment M) at the invariable direction relative to the reference basis I; next follows rotation of vector $L$ with a constant modulus of $\mathrm{HO}$ according to the optimal law determined by equations (3), (8); and, finally, a decrease of the modulus of vector L down to zero with maximum possible velocity (with maximum moment M) at the invariable direction relative to the reference basis I. This program fully determines the spacecraft motion in the process of transfer from the state $\Lambda=\Lambda_{\mathrm{in}}, \omega=0$ to the state $\Lambda=\Lambda_{\mathrm{f}}, \omega=0$, since the equations (1) and the equality $\omega(\mathrm{t})=J^{-1} \mathrm{~L}(\mathrm{t})$ take place.

Since initial and final angular velocities are equal zero and the magnitude of controlling moment is constant $|\mathbf{M}|=$ const $=\mathrm{m}_{0}$, duration of stages of acceleration and braking will be identical. The optimal solution $\omega(\mathrm{t})$ during segment of nominal motion (between acceleration and braking) possesses the properties (7), the vectors $\mathrm{M}$ and $\mathrm{L}$ are orthogonal, the magnitude of angular momentum is maximum and constant $|\mathbf{L}|=$ const $=H_{0}$. Dynamics of motion is fully similar to solution of problem of spacecraft's optimal rotation which was obtained in [1]. If the magnitude $\mathrm{HO}$ of maximum possible angular momentum of a spacecraft is known, then after solving the problem of maximally fast response we shall find the time of a maneuver $\mathrm{T}$; if the time $\mathrm{T}$ of termination of a maneuver is given, then value of parameter $\mathrm{H}_{0}$ in control law is subject to definition.

The optimal control of angular position of a spacecraft can be realized by the method which is similar to the known mode [1]. Since at spacecraft breaking the torque $\mathrm{M}$ is directed strictly against angular momentum $\mathrm{L}$, the instant when braking begins can be predicted with a good accuracy. Duration of rotation damping is $\tau=|\mathbf{L}| / \mathrm{m}_{0}$. The moment of the beginning of braking segment is determined by the condition:

$$
4 \arcsin \frac{K \sqrt{q_{2}^{2}+q_{3}^{2}}}{\sqrt{\left(J_{2} \omega_{2}\right)^{2}+\left(J_{3} \omega_{3}\right)^{2}}}=\frac{K^{2} \sqrt{\omega_{2}^{2}+\omega_{3}^{2}}}{m_{0} \sqrt{\left(J_{2} \omega_{2}\right)^{2}+\left(J_{3} \omega_{3}\right)^{2}}}
$$


Where $q \mathrm{j}$ are the components of quaternion of mismatch

$\tilde{\Lambda}(t) \circ \Lambda_{\mathrm{f}}(\mathrm{j}=0,1,2,3) ; K=|J . \omega|$ is the magnitude of spacecraft's angular momentum. At braking segment, cancellation of angular momentum is make according to linear law $|\mathbf{L}(\mathbf{t})|=\mathrm{H}_{0}-\mathrm{m}_{0}\left(\mathrm{t}-\mathrm{t}_{\mathrm{br}}\right)$ , where $t_{b r}$ is the instant of the beginning of braking.

Determination of the time instant $t_{b r}$ according to actual (measured values) kinematic parameters of motion (angular mismatch and angular velocity) improves the accuracy of guiding the spacecraft to the required state $\Lambda=\Lambda_{\mathrm{f}}, \omega=0$.

\section{Example of mathematical simulation of optimal rotation of a spacecraft}

The aspects of optimization of the terminal control that matches, in the prescribed time $\mathrm{T}$, the right-handed coordinate system $O X Y Z$ fixed to the spacecraft body with the basis whose attitude in the inertial space is given are above investigated. Now, in this section, we describe the numerical finding of the optimal control of the spacecraft slewing maneuver. By way of example, consider the slew maneuver of the spacecraft by $150^{\circ}$ from the initial angular position $\Lambda_{\text {in }}$ in which the spacecraft axes coincide with the axes of the reference basis I to the prescribed angular position $\Lambda_{\mathrm{f}}$. It was assumed in this case that the initial and final angular velocities are zero: $\omega(0)=\omega(\mathrm{T})=0$. The elements of the quaternion $\Lambda_{\mathrm{f}}$ describing the desired attitude of the spacecraft after its slewing are assumed to be $\lambda_{0}=0.258819$, $\lambda_{1}=0.723196, \lambda_{2}=0.5$, and $\lambda_{3}=0.4$. We determine the optimal angular velocity control program $\omega(\mathrm{t})$ for the spacecraft maneuver from the attitude $\Lambda(0)=\Lambda_{\mathrm{in}}$ to the attitude $\Lambda(T)=\Lambda_{\mathrm{f}}$. We assume that the central principal moments of inertia of the spacecraft are $J_{1}=217.3 \mathrm{~kg}-\mathrm{m}^{2}, J_{2}=440.6 \mathrm{~kg}-\mathrm{m}^{2}$, and $J_{3}=416.1$ $\mathrm{kg}-\mathrm{m}^{2}$; maximum magnitude of the controlling moment is $\mathrm{m}_{0}=$ $0.4 \mathrm{~N}-\mathrm{m}$; and maneuver duration is assumed equal $\mathrm{T}=150 \mathrm{~s}$. Notice that the slew quaternion $\Lambda_{\mathrm{s}}$ corresponds to the case when the final rotation vector (Euler's axis) makes approximately the same angle with the axis $O X$ as with the plane perpendicular to $O X$. This is a fairly difficult case of reorientation of a solid body [4].

As a result of solving the boundary value problem corresponding to the transfer of the spacecraft from the attitude $\Lambda(0)=\Lambda_{\text {in }}$ to the attitude $\Lambda(\mathrm{T})=\Lambda_{\mathrm{f}}$, we obtained the rated value for vector $\mathrm{p}(0)=\{0.20548,-0.15337,0.96657\}$ (for possibility of solving the system of the equations (3), (8)). The desired accuracy (approximately 2 angular minutes) is attained at the fifth approximation step of the vector $\mathbf{p}(0)$ to the solution (method of solving the boundary value problem is identical to the algorithm described in [2]).

Key problem is to find of the values $\mathbf{M}(0)$ and $\mathrm{H}_{0}$ at which result of integration of the equations of motion with the initial conditions $\Lambda(0)=\Lambda_{\text {in }}, \omega(0)=0$ satisfies terminal conditions $\Lambda(\mathrm{T})=\Lambda_{\mathrm{f}^{\prime}} \omega(\mathrm{T})=0$ at the instant of time $\mathrm{T}$ taking into account that the entire interval of motion consists of three stages - in the beginning of a maneuver (at acceleration) the trajectory of motion satisfies the equations (1), (10), (11); during spacecraft rotation with constant modulus of angular momentum (between acceleration and braking) the trajectory of motion satisfies the equations (1), (6); in the end of a maneuver (at braking) the trajectory of motion satisfies the equations (1), (10), (12).

The results of mathematical simulation for the slew maneuver under the optimal control are presented in Figure 1-4. Figure 1 shows the dynamics of the optimal angular velocities $\omega_{1}(t), \omega_{2}(t)$, and $\omega_{3}(t)$ in the body-fixed coordinate system in the course of the slew maneuver. One clearly see partition of the entire maneuver into three characteristic stages: acceleration of a spacecraft (gain of angular velocity), spacecraft motion with constant module of angular momentum (segment of nominal rotation), and spacecraft braking (reduction of angular velocity to zero). The law of forming the control also confirms this. The behavior of the rate of changing the angular momentum value is of a relay character, the duration of the segments of acceleration tac (when $\dot{K}>0$ ) and braking $\tau_{\text {br }}$ (when $\dot{K}<0$ ) is identical and equals $\tau=14.6 \mathrm{~s}$. The maximum magnitude of spacecraft angular momentum during optimal rotation (the programmed level) is $\mathrm{H}_{0}=5.83 \mathrm{~N}-\mathrm{m}$-s. The variation of the components $\lambda_{0}(\mathrm{t})$, $\lambda_{1}(\mathrm{t}), \lambda_{2}(\mathrm{t})$, and $\lambda_{3}(\mathrm{t})$ of the quaternion $\Lambda(\mathrm{t})$, which determines the current attitude of the spacecraft in the course of the slew maneuver, is illustrated in Figure 2. Figure 3 shows the plots of the components $l_{1}(\mathrm{t}), l_{2}(\mathrm{t})$, and $l_{3}(\mathrm{t})$ of the ort of angular momentum $\mathrm{L}$ as functions of time. A distinctive feature is that the projection $l_{1}$ varies insignificantly (its variation is much less than the variation of $l_{2}$ and $l_{3}$ ). This indicates that $O X$ is the longitudinal axis of the spacecraft. It is also worth noting that the variables $\omega_{1}(\mathrm{t})$ and $l_{1}(\mathrm{t})$ corresponding to the longitudinal axis of the spacecraft have a constant sign (for all combinations of the boundary values $\Lambda_{\text {in }}$ and $\Lambda_{\mathrm{f}}$ ).

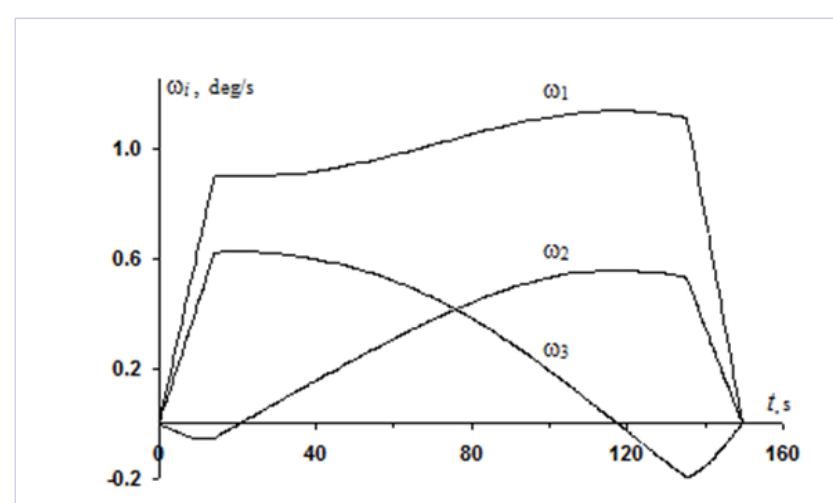

Figure 1: Variation of angular velocities during optimal maneuver 


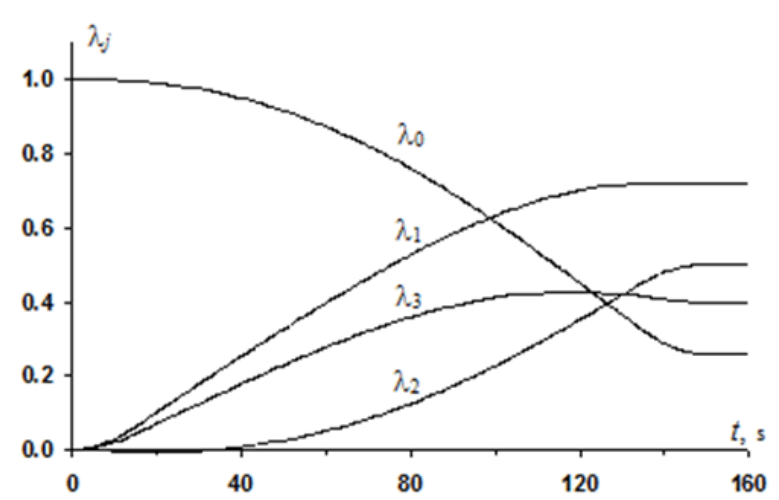

Figure 2: Variation of components of the quaternion $\Lambda$

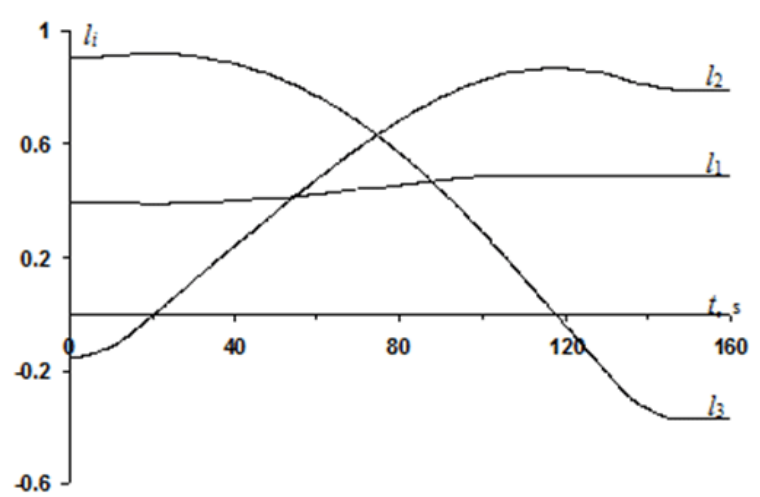

Figure 3: Variation of the variables $11,12,13$ under optimal control

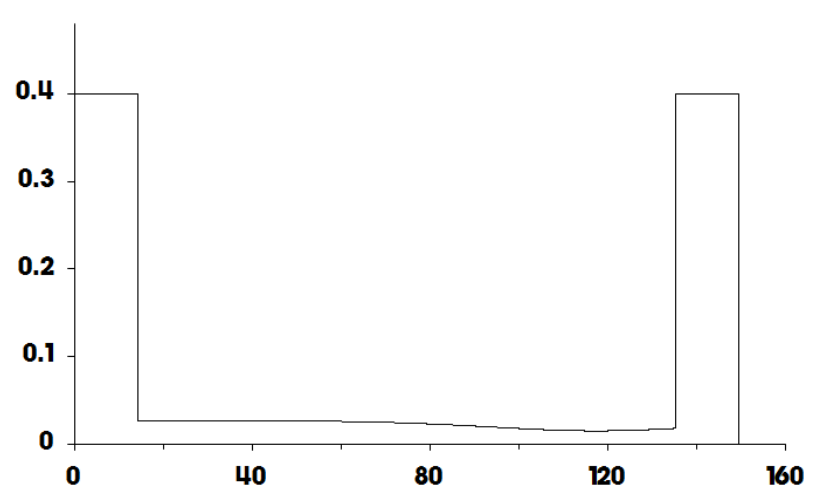

Figure 4: Character of modulus of the controlling moment $\mathrm{M}$ during rotation.

Finally, Figure 4 shows the variation of magnitude of controlling moment in the course of the slew maneuver. All three stages of the maneuver are well pronounced in it. During acceleration and braking processes the controlling moment is maximum possible. In the time interval between acceleration and braking, the controlling moment magnitude is substantially less than during gain and reduction of the spacecraft angular velocity; controlling moment $\mathbf{M}$ is determined according to dynamic Euler's equations in which the time functions of angular velocities $\omega_{\mathrm{i}}(\mathrm{t})$ are a solution to system of equations (3) with expressions (8). At the stages of acceleration and braking, the controlling moment module is constant; during spacecraft rotation with maximum angular momentum the controlling moment magnitude varies slightly (for a dynamically symmetric spacecraft it is constant).

At numerical modelling, the trajectory $\Lambda(\mathrm{t})$ and functions $\omega_{\mathrm{i}}$ corresponding to the optimal maneuver are obtained by integration of the equations of motion (1) and dynamic equations in which the derivatives $\dot{\omega}_{i}$ are calculated according to the equations: (10), (11) if $t<T / 2$ and $|\mathrm{L}|<\mathrm{H}_{0}$; or (6) if $\mathrm{t}_{\mathrm{ac}}$ $\leq \mathrm{t}<\mathrm{t}_{\mathrm{br}} ;$ or (10), (12) if $\mathrm{t} \geq \mathrm{tbr}$, taking into account the initial conditions $\Lambda(0)=\Lambda_{\mathrm{in}}, \omega(0)=0$ and $\mathbf{M}(0)=\mathbf{M}_{\mathrm{st}}$, where $\mathrm{t}_{\mathrm{ac}}$ is the instant of termination of acceleration (the instant of achieving the equality $|\mathrm{L}|=\mathrm{H}_{0}$ in the beginning of maneuver), tbr is the instant of start of braking; $\mathrm{T}$ is the given time of maneuver (the known fixed value); $\mathrm{H}_{0}$ is the calculated constant magnitude of spacecraft's angular momentum between acceleration and braking; tac $\leq \mathrm{T} / 2, \mathrm{tbr} \geq T / 2\left(\Lambda_{\mathrm{in}}\right.$ is the quaternion of attitude before maneuver, $\mathbf{M}_{\mathrm{st}}$ is the calculated value of the controlling moment to start acceleration). The moment of forces $\mathbf{M}$ within the interval between acceleration and braking is calculated by the equations (10) in which angular accelerations $\omega_{i}$ are calculated using the equations (6). In the presented example of simulation, the constants $\mathrm{T}, \mathrm{H}_{0}, t_{a c}, t_{b r}$ and $\mathbf{M}_{\text {st }}$ have the following values: $\mathrm{T}=150 \mathrm{~s}, \mathrm{H}_{0}=5.83 \mathrm{~N}-\mathrm{m}-\mathrm{s}, \mathrm{t}_{\mathrm{ac}}=14.6 \mathrm{~s}, \mathrm{t}_{\mathrm{br}}=135.4 \mathrm{~s}$, and $\mathrm{M}_{\mathrm{st}}=\{0.156484,-0.060996,0.363032\} \mathrm{N}-\mathrm{m}$ (as stated above $\left.\Lambda_{\text {in }}=1\right)$. The values $\mathrm{H}_{0}, t_{a c^{\prime}} t_{b r}$ and $\mathbf{M}_{\mathrm{st}}$ are calculated so that they obtained rotation $\omega(\mathrm{t})$ and the corresponding trajectory $\Lambda(\mathrm{t})$ satisfy the equalities $\Lambda(T)=\Lambda_{\mathrm{f}} \omega(\mathrm{T})=0$ with account for the initial conditions $\Lambda(0)=\Lambda_{\mathrm{in}}, \omega(0)=0$ and $\mathbf{M}(0)=\mathbf{M}_{\text {st }}$.

Accuracy of computations may be improved if to use the law $\mathbf{M}=\tilde{\Lambda} \circ \Lambda_{\text {in }} \circ \mathbf{M}_{\text {st }} \circ \tilde{\Lambda}_{\text {in }} \circ \Lambda$ for mathematical simulation of spacecraft acceleration.

\section{Problem of choosing the optimal time of the slew maneuver}

For a spacecrafts with inertial actuators (powered gyroscopes), is important not only to minimize the angular momentum during the slew maneuver, but also to preset properly (maximum correctly) time T of maneuver ending. The problem consists in determination of such duration of a slew $\mathrm{T}$ (and the value of the parameter $H_{0}$ ), that during the spacecraft motion around its center of mass, evolution of vector $\mathbf{G}$ of the total angular momentum of the system of gyrodynes will not bring this vector beyond the limits of region $\mathrm{S}$ of admissible values (no saturating of the system of gyrodynes will occur), and no necessity will emerge in unloading, i.e., in removal of accumulated angular momentum of the gyrodyne system by applying the moment of forces of another nature (magnetic [4], produced by switching the attitude control jet engines, etc.). Such motions of spacecraft are considered as allowable (in the sense of controlling the spacecraft orientation without unloading the system of gyrodyne). 
At solving the formulated problem (choice of optimal time of slew maneuver) two moments are initial - (a) slew maneuver of a spacecraft occurs according to the method [1], and (b) the stock of the angular momentum of the gy rodynes system should be maximum (at the found parameters of slew maneuver $H_{0}$ and $\mathrm{T}$ ); last requirement allows to reduce probability of involving any other (except the gyrodynes) means of attitude control (for example, jet engines). For the case with zero boundary conditions $\boldsymbol{\omega}(0)=\boldsymbol{\omega}(T)=0$ only a single type of motion is realized: the first phase - acceleration of spacecraft rotation with maximal controlling moment $|\mathbf{M}|=m_{0}$ up to $|\mathbf{L}|=\mathrm{H}_{0}$, next the site of spacecraft motion with constant in magnitude of angular momentum $|\mathbf{L}|=\mathrm{H}_{0}$ (with satisfaction of equalities (7)), and then the symmetric segment of spacecraft braking with maximal controlling moment $|\mathbf{M}|=m_{0}$ up to a full stop of the spacecraft ( $\mathrm{M} \mid \mathrm{L}$ ). Changing the magnitude of angular momentum $\mathrm{G}$ of the system of powered gyroscopes during the slew maneuver is such that the condition $d|\mathbf{G}| d t \approx$ const is true on the segments of acceleration and braking (since the moment $\mathrm{M}$ of the controlling forces is much more perturbations moment Mper). And, in most cases, it is possible to assume $|\mathrm{d}| \mathbf{G}|/ d t|_{t<t a c}=|\mathrm{d}| \mathbf{G}|/ \mathrm{dt}|_{t>t b r}$, where tac is the instant of termination of acceleration; tbr is the instant of the beginning of braking. For the hypothetical case when Mper $=0$, acceleration of spacecraft rotation can be carried out up to situation $|\mathbf{L}|=$ $\mathrm{R}_{0}$, because in this ideal case $|\mathbf{L}|=|\mathbf{G}|$, and within the interval between acceleration and braking we have $d|\mathbf{G}| / \mathrm{dt}=0$ (here $\mathrm{R}_{0}$ is radius of the sphere entered in region $S$ of available values of the angular momentum $\mathrm{G}$ of powered gyroscopes system).

In real conditions of flight $\mathbf{M}_{\mathrm{per}} \neq 0$, and therefore $\mathbf{L}+\mathbf{G} \neq 0$ , and hence in general case $|d| \mathbf{G}|/ d t| \neq 0$ on the segment of nominal rotation (when $|\mathbf{L}(t)|=$ const ). At the presence of perturbations moments $\mathbf{M}_{\text {per }} \neq 0$ there is a problem - what value should be time of a maneuver T (or level H0) that up to termination of maneuver the possible increase in size $|\mathbf{G}|$ was less $\mathrm{R}_{0}-\mathrm{H}_{0}$ (dependence $\mathrm{H}_{0}$ from $\mathrm{T}$ is the monotonously decreasing function). Though perturbations $\mathbf{M}_{\text {per }}$ can "help" the rotation of a spacecraft (in this case $d|\mathbf{G}| / d t<0$ ), but to guarantee such evolution of events throughout all interval of time [tac, tbr] is impossible. Therefore at constructing the designed program of control, we must take into account the worst scenario - to assume the perturbations Mper greatest possible in magnitude and directed against angular momentum $\mathrm{L}$ of spacecraft body. Then $d|\mathbf{G}| / d t|=| \mathbf{M}_{\text {per }} \mid$ and $\max _{t<t} d|\mathbf{G}| / d t=M_{\text {Pcal }}$ is the calculated (the greatest possible) magnitude of perturbation moment $\mathbf{M}_{\text {per }}$ (i.e. $\left|\mathbf{M}_{\text {per }}\right|<\mathbf{M}_{\mathrm{P}_{\text {cal }}}$ ). The increment of magnitude of gyro-system angular momentum $\mathbf{G}$ during spacecraft rotation with $|\mathbf{L}|=$ const will following

$$
\Delta G=\int_{t_{x}}^{t_{\mathrm{x}}}\left|\mathbf{M}_{\mathrm{per}}\right| d t \leq\left(t_{b r}-t_{a c}\right) M_{P_{c a l}}=\left(T-\tau_{a c}-\tau_{b r}\right) M_{P c a l},
$$

Where $\tau_{\mathrm{ac}}$ and $\tau_{\mathrm{br}}$ are the durations of acceleration and braking; $\mathrm{G}=\mid \mathbf{G}$. Thus, the relation

$$
G\left(t_{a c}\right)+\left(T-\tau_{a c}-\tau_{b r}\right) M_{P c a l} \leq R_{0}
$$

should be satisfied, where R0 is the known value beforehand preset.

At the moment of the termination of acceleration $G\left(\tau_{\mathrm{ac}}\right)=\mathbf{L}\left(\tau_{\mathrm{ac}}\right)$ (since $\left.\left|\mathbf{M}_{\mathrm{per}}\right|<<m_{0}\right)$. "Saturation" of system of powered gyroscopes can take place in the limiting case, if the equality $\left|\mathbf{L}\left(\tau_{\mathrm{ac}}\right)\right|=R_{0}-\left(T-\tau_{\mathrm{ac}}-\tau_{\mathrm{br}}\right) M_{\text {Pcal }} \quad$ cal is valid. It is necessary to note, that if the time of slew maneuver $T$ less, then probability of achievement by the moment $t$ br of the braking beginning of the threshold level $\mathbf{G}(t)=R_{0}$ will less, it would seem. However with reduction of time $T$, the required angular momentum $\mathbf{L}\left(\tau_{\mathrm{ac}}\right)$ increases, and the stock $\Delta R=R_{0}-\mathbf{L}\left(\tau_{\mathrm{ac}}\right)$ decreases, that raises probability of presence of gyro-system's "saturation" in the course of slew maneuver (probability of satisfaction of equality $\mathbf{G}=R_{0}$ increases). Here the problem of spacecraft rotation with minimum angular momentum $\mathbf{L}$ during given time $T$ is very important (for that to increase the stock of angular momentum $R_{0}$ - L for its use for indemnification of prospective perturbation moments $\mathbf{M}_{\mathrm{per}}$ ).

At construction of the optimum program of control, we can vary only two parameters - time of a maneuver $T$ or magnitude of spacecraft's angular momentumL $\left(\tau_{\mathrm{ac}}\right)$ (other characteristics are preset by conditions of slew maneuver and cannot be changed). We will write down the equation setting connection between time of rotation $T$, an estimation of the maximum magnitude of perturbation moments $\mathbf{M}_{\mathrm{P} \text { cal }}$, and the computed value of spacecraft's angular momentumL $\left(\tau_{\mathrm{ac}}\right)$ For the rotation of solid body along the trajectory which satisfies the equations (3)-(4), the equality

$$
\int_{0}^{T}|\mathbf{L}| d t=\text { const }=S_{L}
$$

is true, where the value SL is determined exclusively by quaternion of a slew and inertial characteristics of a spacecraft $J_{1^{\prime}} J_{2^{\prime}}$ $J_{3}$ [11]. Having designated $\mathrm{L}_{0}=\left|\mathbf{L}\left(\tau_{\mathrm{ac}}\right)\right|$, and if we believe that on the segments of acceleration and braking, the modulus of angular momentum varies under linear law $|d| \mathbf{L}|/ \mathrm{dt}|=m$, where $\mathrm{m}$ is the maximum speed of varying the modulus of angular momentum, we will receive following relationships:

$$
2 \int_{0}^{T}|\mathbf{L}| d t=L_{0}\left(2 T-\tau_{a c}-\tau_{b r}\right) \text { or } L_{0}\left(T-L_{0} / m\right)=S_{L} \text { (Since } \tau_{\mathrm{br}} \approx \tau_{\mathrm{ac}}=L_{0} / m \text { ). }
$$

Two statements of a problem are possible: (a) at the presence of constraint $T \leq T_{\text {set }}$ we use criterion $\mathrm{L}_{0} \rightarrow \min$ or $\mathrm{M}_{\mathrm{p} \text { cal }} \mathrm{T} \rightarrow \max$ ; and (b) if right endpoint is free, then we receive the system of two equations in which the time of a maneuver $\mathrm{T}$ is specified from the condition $\mathrm{MP}_{\text {cal }} \rightarrow$ max. These equations have the following form

$$
\begin{aligned}
& L_{0}+M_{\mathrm{P} \text { cal }} T=R_{0}, L_{0}\left(T-L_{0} / m\right)=S_{L} \text { or } \\
& T=\left(R_{0}-L_{0}\right) / M_{\mathrm{P} \text { cal, }} L_{0}^{2} / m-L_{0} T+S_{L}=0 ;
\end{aligned}
$$


Whence

$$
L_{0}^{2}\left(1 / M_{\mathrm{Pcal}}+1 / m\right)-L_{0} R_{0} / M_{\mathrm{Pcal}}+S_{L}=0 .
$$

The solution of last equation is

$$
L_{0}=0.5\left(R_{0} m \pm \sqrt{R_{0}^{2} m^{2}-4 S_{L} m M_{\mathrm{Pcal}}\left(m+M_{\mathrm{P} \text { cal }}\right)}\right) /\left(m+M_{\mathrm{Pcal}}\right) \text {. }
$$

If $m \rightarrow \infty$, then values $\tau_{\mathrm{ac}} \rightarrow 0, \tau_{\mathrm{br}} \rightarrow 0$ and $\mathrm{S}_{\mathrm{L}}=\mathrm{L}_{0} \mathrm{~T}$; therefore $\mathrm{R}_{0}-\mathrm{L}_{0}=\mathrm{M}_{\mathrm{P} \text { cal }} \mathrm{T}=\mathrm{MP}$ cal S $\mathrm{L}_{\mathrm{L}} / \mathrm{L}_{0}$, and hence

$$
L_{0}=\left(R_{0} \pm \sqrt{R_{0}^{2}-4 S_{L} M_{\mathrm{Pcal}}}\right) / 2
$$

(It is obvious that $L_{0}<R_{0}$ ).

It is obvious that $\mathrm{m}>\mathrm{M}_{\mathrm{p} \text { cal }}$, and there are two solutions of quadratic equation (in both $L_{0}>0$ ); smaller value $L_{0}$ corresponds to higher border $T\left(T_{\max }\right)$, more big value $\mathrm{L}_{0}$ corresponds to the lower border $\mathrm{T}$ (Tmin, a slew maneuver for minimum time). Time $\mathrm{T}$ we appoint in range $\mathrm{Tmin}<T<T_{\max }$. Outside of interval borders $\left[T_{\text {min' }} T_{\text {max }}\right]$ the rotation without "unloading" of gyro-system is not guaranteed, as "saturation" can occur or because of big $\mathrm{L}_{0}$ which was imparted after acceleration of rotation (it is close to $R_{0}$ ), owing to small time $\mathrm{T}$, or because of the big duration of a stage of nominal rotation (between acceleration and braking), and, as a consequence, the perturbations accumulated up during the slew maneuver "will eat" all stock $R_{0}-L_{0}$ of angular momentum of the gyro-system. Taking into account that $\mathrm{m}>>\left|\mathbf{M}_{\text {per }}\right|$, we will give the approximate estimations (precisely enough reflecting true required levels ${ }_{\mathrm{L} 0}$ and ${ }_{\mathrm{T}}$ ). Since

$$
\sqrt{R_{0}^{2} m^{2}-4 S_{L} m M_{\mathrm{P} \text { cal }}\left(m+M_{\mathrm{P} \text { cal }}\right)} \approx R_{0} m-2 S_{L} m M_{\mathrm{Pcal}} / R_{0},
$$

We can assume as optimum

$$
L_{0} \approx R_{0} / 2-S_{L} M_{\mathrm{Pcal}} / R_{0} \text { and } T \approx 2 S_{L} / R_{0}+R_{0} / 2 m \text {. }
$$

If the magnitude $M_{P c a l}$ is not known or can vary its value over a wide range, then we must appoint time of a maneuver T using the condition of maximum of parameter $M_{P \text { cal }}$ for which the inequality

$$
R_{0}^{2} m>4 S_{L} M_{P \text { cal }}\left(m+M_{P c a l}\right)
$$

is satisfied. The function $S_{L} M_{\text {Pcal }}\left(m+M_{\text {Pcal }}\right)$ increases with increase of $M_{P \text { cal }}$. Hence, it is possible to assume that the roots of quadratic equation in which $L_{0}$ is single unknown value, are converged nearer to each other with increase of the parameter MP cal, and the range $\left[T_{\min }, T_{\max }\right]$ concerning preferable values of the slew maneuver time $T$ will be narrowed. Let us find such critical value of parameter MP cal (we will designate it $M_{\text {crit }}$ ) at which the rotation from position $\Lambda_{\text {in }}$ into position $\Lambda_{\mathrm{f}}$ is still possible without infringement of requirement $|\mathrm{G}| \leq \mathrm{R}_{0}$. For this purpose, it is necessary to solve the equation

$$
R_{0}^{2} m-4 \mathrm{~S}_{\mathrm{L}} \mathrm{M}_{\mathrm{P} \text { cal }}\left(m+M_{\mathrm{P} \text { cal }}\right)=0
$$

With respect to $M_{\mathrm{P} \text { cal }}$ (all other values in this equation are known, they were preset by conditions of the problem of optimal maneuver). As result, we have

$$
M_{\text {crit }}=\left(-m+\sqrt{m^{2}+R_{0}^{2} m / S_{L}}\right) / 2
$$

(Here, $M_{c r i t}=M_{P \max }$ is maximum possible perturbation); usually, $\max \left|\mathbf{M}_{\text {per }}\right|<\mathbf{M}_{\mathrm{P} \max }$ (i.e. $\boldsymbol{M}_{\text {pcal }} \leq \boldsymbol{M}_{P \max }$ ). With account for action of perturbations $\mathbf{M}_{\text {per }}$ having a priori unknown magnitude, by optimal values will be

$$
L_{0}=R_{0} /\left(1+\sqrt{1+R_{0}^{2} / S_{L} m}\right) \text {, and } T=2 S_{L} \sqrt{1+R_{0}^{2} / S_{L} m} / R_{0} .
$$

As a rule, $R_{0}^{2} / S_{L} m<<1$. Therefore, as optimum we can assign Topt $\approx 2 S_{L} / R_{0}$.

Have come to conclusion that $T_{\text {opt }}=2$ Tfast, where Tfast is theoretically achievable lowest level-border of time of spacecraft rotation at the presence of the constraint $|\mathrm{J} \omega| \leq \mathrm{R}_{0}$ (in these formulas $S_{L}=K_{C} t_{p r}$, where tpr is the predicted time of the slew maneuver from the position $\Lambda_{\text {in }}$ into the position $\Lambda_{\mathrm{f}}$ obtained by the simulating the spacecraft motion according to the equations (1), (3), (5) in which $L_{m}=K_{C}>0$ ).

We could expect that in optimal case the time of the slew maneuver should be twice more the time of ideal rotation (when the modulus of spacecraft angular momentum $\mathrm{L}$ is equal to as much as possible admissible value $\mathrm{R} 0$ and the perturbation moments are absent).

\section{Conclusions}

Control of spacecraft attitude using inertial actuators (in particular, by system of powered gyroscopes) is carried out often enough. Efficiency of use of inertial devices (control moment gyroscopes) in regime of spatial rotation is defined by not only the accepted algorithm of spacecraft attitude control but by time of the ending the reorientations maneuver. For the accepting the saturation of gyro-system its total angular momentum should not exceed admissible value (the slew maneuver is carried out due to redistribution of the angular momentum between the system of gyrodynes and spacecraft body). For situation that rotation will occur without the unloading of gyro-system, the modulus of angular momentum of spacecraft body should be obviously less radius of the sphere entered in region of available values of the angular momentum of gyro-system. The reorientation duration in many cases can become critical factor. We have formulated the following problem: it is necessary to define such duration of slew maneuver that during spacecraft motion around the centre of mass the angular momentum vector of gyrodynes system was within region of admissible values, this fact will exclude necessity of "unloading" of gyro- 
system and will provide executing of slew maneuver without the applying of other means for attitude control (for example, jet engines). The solving the specified problem allows to make spatial turns of a spacecraft using only the control moment gyroscopes. The knowledge of fit range of preferable duration of the slew maneuver helps to plan correctly the flight program of spacecraft controlled by the powered gyroscopes, and to appoint correctly time characteristics of main operations (including dynamic operations).

It is known that the method of orientation [1] is optimum at control with use inertial actuators. In the most general case the slew maneuver is partitioned in three characteristic phases: acceleration of the spacecraft (the imparting to it the angular velocity) up to a given magnitude of angular momentum, rotation of the spacecraft with constant modulus of angular momentum, and cancellation of angular velocity down to zero. The problem of attitude control is reduced to solving the three problems: the fastest imparting to a spacecraft the required angular momentum, rotation of the spacecraft with a calculated velocity of motion, and maximum possible deceleration (damping) of the spacecraft. At the segments of acceleration and braking, the controlling moment is maximum possible and parallel to the angular momentum vector, which ensures a minimum time of reaching the present value of the spacecraft angular momentum (or reduction of available angular momentum down to zero). At the stage between acceleration and braking, the spacecraft rotates with angular momentum having constant modulus; the controlling moment is formed using the condition that the spacecraft attitude motion should proceed along the prescribed trajectory of rotation determined by the rated vector of a turn and preset value of the angular momentum modulus. During stages of an intensive gaining and suppressing the angular momentum, the modulus of controlling moment remains to constants. This circumstance allows us to use the described control method of slew maneuver in a case when the controlling moment is limited by sphere. Basing on properties of optimal rotation, the described control method of spacecraft maneuver is exclusively important to apply in cases when the controlling moment is limited by sphere [1].

Optimization of spacecraft reorientation when motion is controlled by inertial actuators (for example, gyrodynes) includes two main tasks. First task is traditional optimization problem which consists in finding the mode of rotation with minimal magnitude of angular momentum. Second problem is new; it consists in calculating the most correct duration of slew maneuver under which necessity of "unloading" of gyro-system is excluded. In this paper, both problems were successfully solved. Numerical example and results of mathematical simulation of optimal spacecraft rotation, as illustration in visual form, are given. They add the made theoretical descriptions and confirm the efficiency and practical feasibility of the control method developed.

\section{Acknowledgements}

The author would like to express greatest thanks to colleagues and specialists from Korolev Rocket Space Corporation (in Russia), and to all participants of the seminars and International conferences on optimization problems.

\section{References}

1. Levskii MV. Some issues of time-optimal control over a spacecraft programmed turn. Cosmic Research. 2011;49(6):521-533.

2. Levskii MV. A special case of spacecraft optimal attitude control. Journal of Computer and Systems Sciences International. 2012;51(4):587-601.

3. Branets V.N., Shmyglevskii I.P. Use of quaternions in problems of orientation of solid bodies. Moscow: Nauka. 1973. [in Russian]

4. Alekseev KB, Bebenin GG. Space vehicles control. Moscow: Mashinostroenie. 1974. [in Russian]

5. Zubov NE. Optimal control over terminal reorientation of a spacecraft based on algorithm with a prognostic model. Cosmic Research. $1991 ; 29(3): 291-300$

6. Ermoshina OV, Krishchenko AP. Synthesis of programmed controls of spacecraft orientation by the method of inverse problem of dynamics. Journal of Computer and Systems Sciences International. 2000;39(2):313-320.

7. Alekseev KB, Malyavin AA, Shadyan AV. Extensive control of spacecraft orientation based on fuzzy logic. Flight. 2009;1:47-53 [in Russian]

8. Van'kov AI. Adaptive robust control of attitude motion of a spacecraft using prognostic models. Cosmic Research. 1994;32(4-5):359-366.

9. Raushenbakh BV, Tokar EN. Spacecraft orientation control. Moscow: Nauka. 1974. [in Russian]

10. Levskii MV. On optimal spacecraft damping. Journal of Computer and Systems Sciences International. 2011;50(1):144-157.

11. Levskii MV. Control of a spacecraft's spatial turn with minimum value of the path functional. Cosmic Research. 2007;45(3):234-247.

12. Levskii MV. A solving the problem of optimal control over turn of a spacecraft within the class of regular motions. Cosmonautics and rocketry-constructing. 1999;(16):22-36 [in Russian]

13. Levskii MV. Method of Controlling a Spacecraft Turn. Russian Federation Patent (Invention's Certificate) No. 2093433. Bulletin "Inventions. Applications and Patents". 1997;(29):271. [in Russian]

14. Kovtun VS, Mitrikas VV. Platonov VN, Revnivykh SG, Sukhanov NA. Mathematical Support for Conducting Experiments with Attitude Control of Space Astrophysical Module Gamma. News from Academy of Sciences USSR. Technical Cybernetics. 1990;(3):144-157 [in Russian]

15. Castruccio PA, Irby .E. All-Digital Attitude Control System for Skylab, in Proc. of the 5th IFAC Symposium on Automatic Control in Space, New York: Pergamon. 1973

16. Sarychev VA, Belyaev MYu, Zykov SG, Sazonov VV, Teslenko VP. Mathematical Models of Processes for Supporting Orientation of the Mir Orbital Station with the Use of Gyrodynes, Preprint of Keldysh Institute of Applied Mathematics (Russian of Academy of Sciences), Moscow. 1989;10. [in Russian]

17. Sarychev VA, Belyaev MYu, Zykov SG, Zueva EYu, Sazonov VV, Saigiraev KhU. Some Problems of Attitude Control of the Orbital Complex Mir Kvant-1 - Kvant-2 (in Proceedings of the 25th Readings Devoted to Elaboration and Development of K.E. Tsyolkovsky's Scientific Heritage. Section "Issues of Rocket and Space Engineering”), Moscow: IIET name of Vavilov S.I. (Russian of Academy of Sciences), 1991;25-32 [in Russian] 